\title{
A Comprehensive Systematic Review and Network Meta-analysis: The Role of anti- angiogenic agents in advanced epithelial ovarian cancer
}

\section{Aya El Helali ( $\square$ ahelali@hku.hk)}

The University of Hong Kong

Charlene HL Wong

The University of Hong Kong

Horace CW Choi

The University of Hong Kong

Wendy WL Chan

The University of Hong Kong

Naomi Dickson

Queen's University Belfast, Northern Ireland

Steven WK Siu

The University of Hong Kong

Karen K Chan

The University of Hong Kong

Hextan YS Ngan

The University of Hong Kong

Roger KC Ngan

The University of Hong Kong

Richard D Kennedy

Queen's University Belfast, Northern Ireland

\section{Research Article}

Keywords: angiogenic agents, epithelial ovarian cancer, platinum-sensitive

Posted Date: November 16th, 2021

DOI: https://doi.org/10.21203/rs.3.rs-1030229/v1

License: (c) (1) This work is licensed under a Creative Commons Attribution 4.0 International License.

Read Full License 
Version of Record: A version of this preprint was published at Scientific Reports on March 9th, 2022. See the published version at https://doi.org/10.1038/s41598-022-07731-1. 


\section{Abstract}

\section{Background}

The efficacy of anti-angiogenic agents (AAAs) in epithelial ovarian cancer (EOC) remains unclear. Therefore, we conducted a systematic review and network meta-analysis (NMA) to synthesize evidence of their comparative effectiveness for improving overall survival (OS) among EOC patients.

\section{Methods}

We searched six databases for randomized controlled trials (RCTs) from their inception to February 2021. We performed a NMA with hazard ratios (HRs) and 95\% confidence intervals (Cls) to evaluate comparative effectiveness among different AAAs in two disease settings, chemotherapy naïve, and recurrent EOC. P-score was used to provide an effectiveness hierarchy ranking. Sensitivity NMA was carried out by focusing on studies that reported high-risk disease chemotherapy-naïve, platinum-resistant, and platinum-sensitive EOC. The primary outcome was OS.

\section{Findings}

We identified 23 RCTs ( $n=11,560$ ) which assessed effectiveness of eight different AAAs. NMA results showed no significant OS difference among anti-angiogenic agents in chemotherapy-naïve. In recurrent EOC, concurrent use of trebananib $(10 \mathrm{mg} / \mathrm{kg}$ ) and chemotherapy was likely to be the best option (P-score: 0.88 , NMA estimate versus chemotherapy: $\mathrm{HR}=1.67,95 \% \mathrm{Cl}$ : 0.94; 2.94$)$. The sensitivity NMA indicated that bevacizumab plus chemotherapy with maintenance treatment (P-score: 0.99 ) and pazopanib plus chemotherapy (P-score: 0.79 ) had the highest probability of being the best intervention for improving OS in high-risk chemotherapy-naïve and platinum-resistant setting, respectively.

Interpretation

AAAs may not play a significant clinical role in chemotherapy-naïve and platinum-sensitive EOC. Conversely, the concurrent use of trebananib with chemotherapy was likely to be the best intervention in recurrent EOC.

\section{Key Points}

1. Anti-angiogenic agents may not play a significant clinical role in chemotherapy naïve, and platinumsensitive EOC.

1. Concurrent use of pazopanib or trebananib with chemotherapy yielded OS benefit in platinumresistant or unclassified recurrent EOC settings, respectively.

2. Bevacizumab delivered concurrently with chemotherapy and maintenance treatment in high-risk chemotherapy naïve setting yielded an improvement in OS. 


\section{Introduction}

Epithelial ovarian cancer (EOC) is the $8^{\text {th }}$ most common female reproductive cancer-related cause of death ${ }^{1}$. The majority of tumors relapse following platinum-based chemotherapy, which is associated with poor outcomes. Despite significant improvements in the management of relapsed high-grade serous ovarian cancer (HGSOC), the treatment options remain limited. Novel strategies have entered the clinic to manage recurrent EOC, including the use of anti-angiogenic therapies and PARP inhibitiors ${ }^{2-6}$.

Approximately $70 \%$ of patients with EOC will eventually relapse following first-line systemic chemotherapy. The fifth Gynecologic Cancer Intergroup (GCIG) recommended re-categorizing platinum sensitivity based on platinum-free interval (PFI) duration ( $<1$ month, 1-6 months, 6-12 months, and >12 months). These subsets corresponded to the previously widely used categories of platinum-refractory, platinum-resistant, partially platinum-sensitive, and fully platinum-sensitive, attempting to standardize second-line therapy ${ }^{7,8}$. Patients with a $\mathrm{PFI}<6$ months have fewer alternative treatment options and consequent poorer survival outcomes ${ }^{9,10}$.

Anti-angiogenic agents have attained regulatory approval to manage a diverse spectrum of solid cancers. Angiogenesis is a key pathological ${ }^{11-15}$ feature of EOC, and anti-angiogenic agents have dominated the field of drug development in EOC, particularly in the setting of recurrent disease. In EOC, bevacizumab is the only anti-angiogenic agent approved by the Food and Drug Administration (FDA) and European Medicines Agency (EMA). The FDA approved bevacizumab in platinum-resistant EOC ${ }^{2}$ and platinumsensitive EOC ${ }^{3,16}$ combined with chemotherapy. Additionally, the EMA has approved bevacizumab's clinical use in EOC irrespective of disease state: chemotherapy-naïve ${ }^{17,18}$; platinum-sensitive ${ }^{3}$ and platinum-resistant ${ }^{2}$ disease.

The approval of other anti-angiogenic agents by the regulatory authorities has been more challenging, with many awarded orphan designations until they receive marketing approval. The FDA and EMA assigned orphan designation for trebananib and cediranib to manage recurrent EOC, combined with chemotherapy. There is variation in the recommendations for the appropriate use and disease setting for anti-angiogenic agents in EOC. The National Comprehensive Cancer Network (NCCN) ${ }^{19}$ and the ESMOESGO Ovarian Cancer Consensus Conference Working Group ${ }^{20}$ advocate for the clinical use of bevacizumab in both the chemotherapy-naïve setting and the recurrent disease setting. However, the National Institute for Health and Care Excellence (NICE) ${ }^{21}$ does not support the clinical use of bevacizumab in advanced EOC. Additionally, several meta-analyses were published to shed light on this clinical knowledge gap. Unfortunately, these studies further demonstrate the clinical uncertainty and show inconsistency in reporting regarding the clinical efficacy of anti-angiogenic agents in the EOC disease state spectrum ${ }^{22-31}$ (Supplementary Table 2). We, therefore, a systematic review and network meta-analysis (NMA) to synthesize evidence of comparative effectiveness of different anti-angiogenic agents for improving overall survival (OS) among EOC patients 


\section{Methods}

We performed a systematic review and NMA of the curated literature according to the Preferred Reporting Items for Systematic Reviews and Meta-Analyses (PRISMA) extension statement for $\mathrm{NMA}^{32}$. A prospective protocol was registered in PROSPERO (registration: CRD42021240133) ${ }^{33}$.

\section{Eligibility criteria}

To be eligible for inclusion in this review, a randomized controlled trial (RCT) should be published in English and satisfy the following criteria: patient, Intervention, comparison, and Outcome (PICO). Patients with either locally advanced or recurrent EOC were included. In addition, as defined in current evidencebased guidelines, anti-angiogenic agents, compared with placebo or any types of standard of care chemotherapy, were considered eligible. Included RCTs should report hazard ratio (HR) and 95\% confidence intervals $(\mathrm{Cl})$ for the outcomes of overall survival (OS) or progression-free survival (PFS) in both intervention and control groups. The primary outcome of this systematic review was OS, while the secondary outcome was PFS.

\section{Literature search}

A systematic search was undertaken to identify RCTs using the following databases from inception to February 2021: EMBASE; PubMed; Ovid Medline; Cochrane Central Register of Controlled Trials (CCTR); Cochrane Database of Systematic Reviews (CDSR); and ClinicalTrials.gov (www.clinicaltrials.gov), ASCO and ESMO abstract database. Furthermore, we searched references of relevant articles retrieved from the electronic search for additional citations. No restrictions on publication status were imposed. The key search terms used were "ovarian cancer", "ovarian neoplasm" [MESH Term], "anti angiogenic agents", "angiogenesis inhibitors", "Bevacizumab", "Nintedanib", "Pazopanib", "Cediranib", "Trebananib", "Sorafenib", "platinum sensitive ovarian cancer", "platinum resistant ovarian cancer", "VEGF", "VEGFR", "PDGF", "PDGFR", "FGF", "FGFR", "TIE", "RET", "AXL", "FLT" and "FLT-3".

\section{Study selection, data extraction, and quality assessment}

After a comprehensive literature search, we used reference management software (EndNote) to identify and remove potential duplicate RCTs. Two review authors (AE, WLC) independently screened titles and abstracts of retrieved RCTs and assessed full texts for eligibility. Discrepancies were resolved through consensus; unsolved discrepancies were settled via consulting a third review author (ND). In the event of duplicate publications, priority was given to the publication reporting the most extended follow-up associated with our primary and secondary outcomes. To be included in NMA, RCTs should share a common comparator that served as a bridge for indirect comparison of different anti-angiogenic agents.

Two review authors ( $\mathrm{AE}, \mathrm{HC}$ ) collected the following information from each included RCT using an established data abstraction method: year of publication, country, disease settings, number of patients randomized, patient demographics, details of interventions and comparators, results of prespecified 
outcomes, and study funding sources. Data extracted was further independently evaluated by one author $(\mathrm{CW})$.

Two review authors (AE, WLC) assessed the risk of bias of each eligible RCT independently using the Cochrane risk of bias tool $2^{34}$. The following domains were assessed: bias arising from the randomization process; bias due to deviations from the intended interventions; bias due to missing outcome data; bias in the measurement of the outcome; and bias in selecting the reported results ${ }^{36}$. The overall risk of bias of each trial was judged as low, some concerns, or high risk of bias ${ }^{36}$. Disagreements were resolved through consensus; unsolved disagreements were settled via consulting a third review author $(\mathrm{HC})$.

\section{Data Analysis}

NMA

NMA offered a set of methods to visualize and interpret a broader picture of current evidence and assessed comparative effectiveness among various interventions ${ }^{35}$. It provided indirect evidence (estimates between various interventions via common comparators) when direct evidence (head-to-head estimates of various interventions) was not available ${ }^{36}$. With the common comparators, we conducted indirect comparisons between different interventions on the primary and secondary outcomes. In this systematic review, NMA was performed to investigate the "relatively most" effective anti-angiogenic agent for improving OS and PFS among EOC patients in two disease settings, namely i) chemotherapy naïve, ii) recurrent EOC.

A network plot was generated for each disease setting to show all interventions included in the NMA ${ }^{37,38}$. Comparative effectiveness results of all possible pairs of comparisons were summarized with an HR and $95 \% \mathrm{Cl}^{37,38}$. P-score was used to provide an effectiveness hierarchy ranking ${ }^{37,38}$. A higher P-score represents superior performance ${ }^{39}$.

Given the potential impact of anti-angiogenic agents in i) high-risk group in the chemotherapy naïve setting ${ }^{40}$, ii) platinum-resistant and iii) platinum-sensitive groups in recurrent EOC setting, sensitivity analyses of NMA were carried out by only including studies that explored the effectiveness of antiangiogenic agents on primary and secondary outcomes in these three group of patients within the corresponding disease settings. High-risk disease was defined as FIGO stage IV or inoperable stage or sub-optimally ( $>1 \mathrm{~cm}$ residual disease) resected FIGO stage $\mathrm{III}^{41}$.

Consistency of direct and indirect evidence on the same comparison was a fundamental assumption of $\mathrm{NMA}^{42}$. Therefore, the separating indirect from direct evidence (SIDE) approach using the backcalculation method was performed for each comparison in the network, on which both direct and indirect evidence were available, to evaluate evidence consistency ${ }^{43}$. The inconsistency with the p-value less than 
0.05 was considered a significant concern. NMA was conducted using the "netmeta" package in $\mathrm{R}$

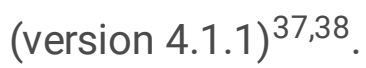

Assessment of Publication bias

Publication bias on the primary outcome in each disease setting was assessed via a comparisonadjusted funnel plot produced by $\mathrm{R}$ (version 4.1.1) ${ }^{37,38}$ when at least ten studies were included in NMA ${ }^{34}$.

\section{Results}

\section{Study selection and characteristics of included RCTs}

A total of 2,363 publications were identified through the initial literature search, and 1563 studies remained after duplications were excluded. Following the title and abstract screen, 1,392 publications were removed because they did not meet the study's hypothesis or were abstracts of full-text publications included in eligible articles review. One hundred seventy-one potentially relevant articles were identified for the comprehensive review. Following this process, 23 multicentre phase II-III RCTs were analyzed in this NMA. Details of literature search and selection for RCTs are presented in Supplementary Fig 1.

Characteristics of the 23 included RCTs are summarized in supplemtary table 1 . They included a total of 11,560 patients, with sample sizes varying from 84 to 1873 . Four RCTs were conducted in the chemotherapy naïve setting, three in the first-line maintenance setting, and 16 in the recurrent EOC setting. Eight different anti-angiogenic agents administered concurrently with chemotherapy, with or without maintenance treatment, were evaluated in all included trials, while the standard of care chemotherapy was reported as control intervention in 19 trials. All RCTs assessed OS and PFS, except that Duska et al. ${ }^{44}$ and the East Asian Study ${ }^{45}$ trials reported PFS only. Amongst these 23 RCTs, the overall risk of bias for 12 RCTs (52.2\%) was rated as low and 11 (47.8\%) as having some concerns (Supplementary table 3).

\section{NMA results}

\section{Chemotherapy naïve setting}

For improving OS and PFS, each network included one three-arm trial and three two-arm trials (Fig 1a, 1b, respectively). The standard of care chemotherapy in these networks was carboplatin combined with paclitaxel (Car/Pac). NMA results suggested no significant difference in OS and PFS among the concurrent use of anti-angiogenic agents and the standard of care chemotherapy with or without maintenance treatment (Fig 1C, 1d). Effectiveness hierarchy ranking results of the interventions are shown in Supplementary Fig 2. As the inconsistency p-values for the two comparisons in OS and PFS were 0.75 and 0.31 , respectively (Fig $1 \mathrm{e}, 1 \mathrm{f}$ ), there was no significant inconsistency in these two NMA. 
There were four RCTs in the high-risk group of chemotherapy naïve setting. The ICON7 trial defined the high-risk disease as FIGO stage IV, inoperable, or sub-optimally ( $>1 \mathrm{~cm}$ residual disease) resected FIGO stage $1 \mathrm{II}^{41}$. These criteria were shared with the TRINOVA-3 ${ }^{46}$, GOG-0218 trials ${ }^{47}$, and the AGO-OVAR $12^{48}$. Results of sensitivity analysis of NMA supported the use of bevacizumab, administered concurrently with chemotherapy and maintenance treatment (Bev_CM), with an improved OS (P-score: 0.99, NMA estimate of Bev_CM versus Car/Pac: HR= 0.82, 95\% Cl: 0.70; 0.97) (Fig 2a, 2b) and PFS (P-score: 0.99, NMA estimate of Bev_CM versus Car/Pac: HR= $0.68,95 \% \mathrm{Cl}: 0.59 ; 0.79)$ (Fig 2c, 2d). It was likely to be the best treatment option for both outcomes for this group of patients. Effectiveness hierarchy ranking results of the interventions for OS and PFS are shown in figure $2 \mathrm{e}$, and $2 \mathrm{f}$, respectively.

\section{Recurrent EOC setting}

For improving OS, the network included one three-arm trial and 13 two-arm trials (Fig 3a). The concurrent use of trebananib $(10 \mathrm{mg} / \mathrm{kg})$ and chemotherapy (Tre_C10) was likely to be the best option (P-score: 0.88 , NMA estimate of Standard of care chemotherapy (Chemo) versus Tre_C10: HR= 1.67, 95\% Cl: 0.94; 2.94), followed by sorafenib combined with chemotherapy and maintenance treatment (Sor_CM) (P-score: 0.87, NMA estimate of Chemo versus Sor_CM: HR= 1.54, 95\% Cl: 1.07; 2.21) (Fig 3b).

For improving PFS, the network included one three-arm trial and 14 two-arm trials (Fig 3c). The combination of chemotherapy and maintenance treatment with bevacizumab (Bev_CM) demonstrated more significant improvement when compared to standard of care chemotherapy $(\mathrm{HR}=0.48,95 \% \mathrm{Cl}: 0.33$; 0.70) (Fig 3d). It was also likely to be the most effective option (P-score: 0.89 ), followed by the concurrent use of Bevacizumab and chemotherapy (Bev_C) (P-score: 0.82, NMA estimate of Bev_C versus Chemo: $\mathrm{HR}=0.54,95 \% \mathrm{Cl}: 0.44 ; 0.67)$. Effectiveness hierarchy ranking results of the interventions for OS and PFS are shown in figure $3 e$, and $3 f$, respectively.

\section{Platinum resistant group}

There were three RCTs in the platinum-resistant group of recurrent EOC setting. Results of sensitivity analysis of NMA showed that the concurrent use of pazopanib and chemotherapy (Paz_C) had the highest probability of being the best intervention for improving OS (P-score: 0.79, NMA estimate of Chemo versus Paz_C: $\mathrm{HR}=1.67,95 \% \mathrm{Cl}: 0.89 ; 3.13)$, followed by sorafenib combined with chemotherapy and maintenance treatment (Sor_CM) (P-score: 0.76, NMA estimate of Chemo versus Sor_CM: HR= 1.54, $95 \%$ Cl: $1.07 ; 2.21$ ) (Fig 4a, 4b). The former intervention (Paz_C) was also likely to be the most effective intervention for improving PFS ( $p$-score: 0.85 , NMA estimate of Chemo versus Paz_C: $\mathrm{HR}=2.38,95 \% \mathrm{Cl}$ : $1.43 ; 3.96)$ (Fig 4c, 4d). Effectiveness hierarchy ranking results of the interventions for OS and PFS are shown in figure $4 \mathrm{e}$, and $4 \mathrm{f}$, respectively

\section{Platinum sensitive group}

There were four RCTs in the platinum-sensitive group of recurrent EOC setting. Sensitivity analysis of NMA results showed no significant difference in OS among the use of anti-angiogenic agents 
and standard of care chemotherapy (Fig 5a, 5b). Nonetheless, the combination of chemotherapy and maintenance treatment with i) bevacizumab (Bev_CM) and ii) cediranib (Ced_CM), as well as iii) bevacizumab combined with chemotherapy alone (Bev_C) were significantly more effective than chemotherapy in improving PFS, with HRs of 0.48 (95\% Cl: $0.36 ; 0.66), 0.56$ (95\% Cl: $0.41 ; 0.77)$ and 0.58 $(95 \% \mathrm{Cl}: 0.47 ; 0.70)$ respectively (Fig $5 \mathrm{c}, 5 \mathrm{~d}$ ). Amongst these interventions, bevacizumab combined with chemotherapy and maintenance treatment (Bev_CM) had the highest probability of being the best option for prolonging PFS ( $p$-score: 0.85 ). Effectiveness hierarchy ranking results of the interventions for OS and PFS are shown in figure $5 e$, and $5 f$, respectively

\section{Publication bias assessment}

Based on the visual inspection of comparison-adjusted funnel plot of 15 included RCTs on the primary outcome of OS in the recurrent EOC setting, there was no evidence of funnel plot asymmetry (Egger's test: $p=0.8347$, Supplementary Fig 3), indicating absence of publication bias. However, for other NMAs on the OS outcome in chemotherapy naïve, publication bias could not be assessed due to insufficient number of included studies.

\section{Discussion}

To our knowledge, this is the most comprehensive NMA which explores the clinical impact of antiangiogenic agents in EOC. We demonstrate the probable lack of efficacy of anti-angiogenic agents in non-high-risk chemotherapy naïve, and platinum-sensitive EOC. Furthermore, in the absence of a predictive biomarker for response to anti-angiogenic agents, we highlight their probable positioning in managing high-risk chemotherapy naïve and platinum-resistant EOC.

The variation in OS outcomes reported in the high-risk chemotherapy naïve disease setting may make it difficult to infer any recommendations regarding the role of anti-angiogenic agents in this disease setting. Data interpreted of the ICON7 trial demonstrated that high-risk disease is associated with an OS benefit $(p=0 \cdot 01 \text {, HR: } 0 \cdot 78 ; 95 \% \mathrm{Cl}: 0 \cdot 63-0 \cdot 97)^{49}$. Conversely, the AGO-OVAR 12 study reported that OS in the highrisk group did not favor nintedanib (HR: $1 \cdot 14 ; 95 \% \mathrm{Cl}: 0 \cdot 89-1 \cdot 45$ ) ${ }^{48}$. Additionally, the GOG-0218, FIGO stage IV disease favored the concurrent bevacizumab and chemotherapy followed by bevacizumab maintenance arm (HR: 0.72; 95\% Cl: 0.53-0.97) ${ }^{50}$. When used concurrently with chemotherapy followed by maintenance until progression, we demonstrate that bevacizumab was associated with the highest probability of OS (Fig 2b) and PFS (Fig 2d) benefit in high-risk chemotherapy naïve EOC. Furthermore, we demonstrate that anti-angiogenic agents may not play a role in managing non-highrisk chemotherapy naïve EOC.

Interestingly, primary platinum resistance was associated with significant PFS and OS benefit from adding anti-angiogenic agents to chemotherapy. In addition, we identified that in the setting of platinumresistant disease, Pazopanib (P-score $=0.79$ ) and Sorafenib (P-score $=0.76$ ), administered concurrently with chemotherapy, resulted in clinically significant improvements in OS (Fig 4). 
The FDA defines clinical outcomes as a direct measure of benefit from an intervention in a trial, while a surrogate end-point is used as a predictive substitute for clinical benefit. Nevertheless, surrogate outcome measures play a notable role in solid cancers ${ }^{51}$. PFS is the most commonly used primary end-point and surrogate marker for OS in solid cancer ${ }^{52,53}$, and the prevalence of its use is growing. The FDA has approved many cancer drugs based on surrogate end-point data. Among those receiving regulatory approval, $57 \%$ of cancer drugs did not demonstrate an 0 S benefit ${ }^{54}$.

Furthermore, Pasalic et al. and Prasad et al. highlight the suboptimal predictive value of PFS as a surrogate for $\mathrm{OS}^{55,56}$. Additionally, in EOC, the predictive value of PFS was further refuted as a surrogate for $\mathrm{OS}^{57}$. Furthermore, interpretation of the result findings presented in this NMA show that PFS falls short of being a substitute for OS in EOC. Therefore, we consider that the findings presented in this NMA are collectively significant and highlight the importance of selecting the appropriate EOC patient group, high-risk, and platinum resistant disease, that are likely to derive OS benefit from anti-angiogenic agents. Consequently, regulators need to cautiously interpret trials that report PFS benefits without mature OS data.

To date, there are no clinically approved predictive molecular biomarkers that may have clinical utility in selecting the subtype of patients likely to benefit from anti-angiogenic agents. However, there is a clear clinical unmet need. Nevertheless, there has been a significant drive to identify patients who benefit from bevacizumab. Bentink et al. identified molecular subtypes (split $1-4$ ) in HGSOC, generated from 129 FFPE patient tumor samples. Split 1 was associated with angiogenesis and extracellular matrix proteins, termed the angiogenic subtype, corresponding with the Tothill C1 and TCGA mesenchymal subtypes ${ }^{58}$.

Backen et al. identified that high Ang1/low Tie2 serum values were associated with significantly improved PFS for bevacizumab-treated patients in the ICON7 trial (median, 23.0 months vs. 16.2; $\mathrm{p}=$ $0.003)^{59}$. In addition, Gourley et al. developed a gene signature to identify angiogenic molecular subtypes in ovarian cancer ${ }^{60}$. These findings were validated in the ICON7 trial and demonstrated that bevacizumab had a favorable PFS impact on patient tumors harboring the angiogenic subtype ${ }^{60}$.

Collinson et al. identified three potentially predictive biomarkers, mesothelin, fms-like tyrosine kinase-4 (FLT4), and $a_{1}$-acid glycoprotein (AGP), from serum samples of patients recruited to the ICON7 trial.

These biomarkers identified the subgroup of patients likely to benefit from bevacizumab, predominantly leading to an improvement in median PFS of 5.5 months in the signature positive subgroup $(p=0.001))^{61}$. In the GOG218 trial, Birrer et al. demonstrated a correlation between microvessel density (MVD), tumor VEGF-A (tVEGF-A) expression, and survival outcome in the bevacizumab arm. Comparing chemotherapy with bevacizumab versus chemotherapy alone, higher MVD showed predictive value for PFS $(p=0.018)$ and OS $(p=0.0069)$. tVEGF-A expression showed potential predictive value for OS (and PFS) $(p=0.023)^{62}$. However, the results described above need to be independently validated in more extensive trials before clinical practice implementation. 
We identified a few limitations of this study. It is essential to highlight that the small sample size in the MITO-11 trial ${ }^{63}$ and the TRIAS trial ${ }^{64}$ may impact the interpretation of the OS results illustrated in Fig 4. Therefore, making it challenging to definitively assess the impact of pazopanib on OS in platinumresistant disease. Furthermore, not all the trials identified in the recurrent disease setting reported separate PFS and OS based on PFI. This, therefore, reduced the number of trials included in the sensitivity analysis for each pre-defined PFI cohort

Following our observations, we suggest a potential treatment approach (Supplementary Fig 4) for EOC. In high-risk treatment naïve disease, bevacizumab combined with standard chemotherapy and followed maintenance may provide the best PFS and OS. On recurrence, anti-angiogenic agents, particularly pazopanib in combination with chemotherapy, may be most appropriate in managing platinum-resistant EOC. In contrast, PARP inhibitors combined with anti-angiogenic agents may play a more critical role than anti-angiogenic agent monotherapy in platinum-sensitive $\mathrm{EOC}^{65}$. The level IA evidence presented in this study further emphasizes the need for better patient selection for anti-angiogenic agents in EOC.

\section{Declarations}

\section{Acknowledgements:}

\section{Author Disclosures.}

- Professor Richard Kennedy is employed by Almac as the Medical Director. RDK

- No author disclosures for the remaining authors: AE, CW, HC, WLC, ND, SWKS, KKC, HYSN, RKCN

\section{Author contributions.}

- Conceptualization: AE

- Methodology: AE, CW, HC, WLC, ND, RDK

- Formal analysis: $\mathrm{AE}, \mathrm{CW}, \mathrm{HC}$

- Writing - original draft: $A E$

- Writing - review \& editing: AE, CW, HC, WLC, ND, SWKS, KKC, HYSN, RKCN, RDK

Funding: This research did not receive any specific grant from funding agencies in the public, commercial, or not-for-profit sectors

\section{References}

1. World Health Organization. Globocan 2020. Int. Agency Res. (2020). 
2. Pujade-Lauraine, E. et al. Bevacizumab combined with chemotherapy for platinum-resistant recurrent ovarian cancer: The AURELIA open-label randomized phase III trial. J. Clin. Oncol. 32, 1302-8 (2014).

3. Aghajanian, C. et al. OCEANS: a randomized, double-blind, placebo-controlled phase III trial of chemotherapy with or without bevacizumab in patients with platinum-sensitive recurrent epithelial ovarian, primary peritoneal, or fallopian tube cancer. J. Clin. Oncol. 30, 2039-45 (2012).

4. Coleman, R. L. et al. Bevacizumab after bevacizumab in platinum-sensitive recurrent ovarian cancer: A subgroup analysis of GOG0213. J. Clin. Oncol. 34, (2016).

5. J.A., L. et al. Randomised double-blind phase III trial of cediranib (AZD 2171) in relapsed platinum sensitive ovarian cancer: Results of the ICON6 trial. Eur. J. Cancer 49, S5-S6 (2013).

6. Ledermann, J. et al. Olaparib Maintenance Therapy in Platinum-Sensitive Relapsed Ovarian Cancer. New England Journal of Medicine vol. 366 1382-1392 (2012).

7. Wilson, M. K. et al. 5th Ovarian Cancer Consensus Conference of the Gynecologic Cancer InterGroup: Recurrent Disease. Ann. Oncol. mdw663 (2016) doi:10.1093/annonc/mdw663.

8. Friedlander, M. et al. Clinical trials in recurrent ovarian cancer. Int. J. Gynecol. Cancer 21, 771-775 (2011).

9. Cannistra, S. A. Cancer of the Ovary. N Engl J Med 35124351, 2519-29 (2004).

10. Naumann, R. W. \& Coleman, R. L. Management strategies for recurrent platinum-resistant ovarian cancer. Drugs 71, 1397-1412 (2011).

11. Folkman, J. \& Folkman, J. Angiogenesis: an organizing principle for drug discovery? Nat. Rev. Drug Discov. 6, 273-86 (2007).

12. Oklu, R., Walker, T. G., Wicky, S. \& Hesketh, R. Angiogenesis and current anti-angiogenic strategies for the treatment of cancer. Journal of Vascular and Interventional Radiology vol. 21 1791-1805 (2010).

13. Folkman, J. Tumor angiogenesis: therapeutic implications. N. Engl. J. Med. 285, 1182-1186 (1971).

14. Bergers, G. \& Benjamin, L. E. Tumorigenesis and the angiogenic switch. Nat. Rev. Cancer 3, 401410 (2003).

15. Carmeliet, P. \& Jain, R. K. Molecular mechanisms and clinical applications of angiogenesis. Nature 473, 298-307 (2011).

16. Coleman, R. L. et al. Bevacizumab and paclitaxel-carboplatin chemotherapy and secondary cytoreduction in recurrent, platinum-sensitive ovarian cancer (NRG Oncology/Gynecologic Oncology 
Group study G0G-0213): A multicentre, open-label, randomised, phase 3 trial. The Lancet Oncology (2017) doi:10.1016/S1470-2045(17)30279-6.

17. Perren, T. J. et al. A phase 3 trial of bevacizumab in ovarian cancer. N. Engl. J. Med. 365, 2484-96 (2011).

18. Burger, R. a et al. GOG 218 - Incorporation of bevacizumab in the primary treatment of ovarian cancer. N. Engl. J. Med. 365, 2473-83 (2011).

19. Cancer, O. NCCN.org NCCN Guidelines for Patients ${ }^{\circledR}$ available at www.nccn.org/patients NCCN Clinical Practice Guidelines in Oncology (NCCN Guidelines $\AA$ ). www.nccn.org/patients (2021).

20. Colombo, N. et al. ESMO-ESGO consensus conference recommendations on ovarian cancer: Pathology and molecular biology, early and advanced stages, borderline tumours and recurrent disease. Ann. Oncol. 30, 672-705 (2019).

21. Managing advanced (stage II-IV) ovarian cancer - NICE Pathways. https://pathways.nice.org.uk/pathways/ovarian-cancer\#path=view\%3A/pathways/ovariancancer/managing-advanced-stage-ii-iv-ovarian-cancer.xml\&content=view-node\%3Anodes-second-lineand-subsequent-chemotherapy.

22. Wang, H., Xu, T., Zheng, L. \& Li, G. Angiogenesis Inhibitors for the Treatment of Ovarian Cancer: An Updated Systematic Review and Meta-analysis of Randomized Controlled Trials. International Journal of Gynecological Cancer (2018) doi:10.1097/IGC.0000000000001258.

23. Wu, Y. S., Shui, L., Shen, D. \& Chen, X. Bevacizumab combined with chemotherapy for ovarian cancer: an updated systematic review and meta-analysis of randomized controlled trials. Oncotarget $\mathbf{8}$, 10703-10713 (2017).

24. Marchetti, C. et al. Efficacy and toxicity of bevacizumab in recurrent ovarian disease: An update meta-analysis on phase III trials. Oncotarget 7, 13221-13227 (2016).

25. Jiang, Y., Sun, X., Kong, B. \& Jiang, J. Antiangiogenesis therapy in ovarian cancer patients: An updated meta-analysis for 15 randomized controlled trials. Medicine (Baltimore). (2018) doi:10.1097/MD.0000000000011920.

26. Li, J., Zhou, L., Chen, X. \& Ba, Y. Addition of bevacizumab to chemotherapy in patients with ovarian cancer: a systematic review and meta-analysis of randomized trials. Clin. Transl. Oncol. 17, 673-683 (2015).

27. Zhou, M., Yu, P., Qu, X., Liu, Y. \& Zhang, J. Phase III trials of standard chemotherapy with or without bevacizumab for ovarian cancer: A meta-analysis. PLoS One 8, (2013). 
28. Ye, Q. \& Chen, H. L. Bevacizumab in the treatment of ovarian cancer: A meta-analysis from four phase III randomized controlled trials. Arch. Gynecol. Obstet. 288, 655-666 (2013).

29. Li, J., Li, S., Chen, R., Yu, H. \& Lu, X. The prognostic significance of anti-angiogenesis therapy in ovarian cancer: a meta-analysis. J. Ovarian Res. (2015) doi:10.1186/s13048-015-0181-z.

30. Li, X., Zhu, S., Hong, C. \& Cai, H. Angiogenesis inhibitors for patients with ovarian cancer: A metaanalysis of 12 randomized controlled trials. Curr. Med. Res. Opin. 32, 555-562 (2016).

31. Wang, T. S. et al. A meta-analysis of bevacizumab combined with chemotherapy in the treatment of ovarian cancer. Indian J. Cancer 51, e95-e98 (2014).

32. Hutton, B. et al. The PRISMA extension statement for reporting of systematic reviews incorporating network meta-analyses of health care interventions: checklist and explanations. Ann. Intern. Med. 162, 777-784 (2015).

33. Booth, A. et al. The nuts and bolts of PROSPERO: An international prospective register of systematic reviews. Syst. Rev. (2012) doi:10.1186/2046-4053-1-2.

34. Higgins, J., Savović, J., Page, M. J. \& Sterne, J. A. C. RoB 2: A revised Cochrane risk-of-bias tool for randomized trials. British Medical Journal (2019).

35. Lu, G. \& Ades, A. E. Combination of direct and indirect evidence in mixed treatment comparisons. Stat. Med. 23, 3105-3124 (2004).

36. Mills, E. J., Thorlund, K. \& loannidis, J. P. A. Demystifying trial networks and network meta-analysis. BMJ 346, (2013).

37. Neupane, B., Richer, D., Bonner, A. J., Kibret, T. \& Beyene, J. Network meta-analysis using R: a review of currently available automated packages. PLoS One 9, e115065 (2014).

38. Rucker, G., Krahn, U., Konig, J., Efthimiou, O., Papakonstantinou, T., \& Schwarzer, G. Package ‘ netmeta '. Network Meta-Analysis using Frequentist Methods. (2021) doi:10.1007/978-3-319-21416.

39. Rücker, G. \& Schwarzer, G. Ranking treatments in frequentist network meta-analysis works without resampling methods. BMC Med. Res. Methodol. 15, 58 (2015).

40. Monk, B. J., Minion, L. E. \& Coleman, R. L. Anti-angiogenic agents in ovarian cancer: Past, present, and future. Ann. Oncol. (2016) doi:10.1093/annonc/mdw093.

41. Colomban, O. et al. Bevacizumab for Newly Diagnosed Ovarian Cancers: Best Candidates Among High-Risk Disease Patients (ICON-7). JNCl Cancer Spectr. 4, (2020).

42. Veroniki, A. A., Vasiliadis, H. S., Higgins, J. P. T. \& Salanti, G. Evaluation of inconsistency in networks of interventions. Int. J. Epidemiol. 42, 332-345 (2013). 
43. van Valkenhoef, G., Dias, S., Ades, A. E. \& Welton, N. J. Automated generation of node-splitting models for assessment of inconsistency in network meta-analysis. Res. Synth. Methods 7, 80-93 (2016).

44. Duska, L. R. et al. A randomized phase Il evaluation of weekly gemcitabine plus pazopanib versus weekly gemcitabine alone in the treatment of persistent or recurrent epithelial ovarian, fallopian tube or primary peritoneal carcinoma. in Gynecologic Oncology (2020). doi:10.1016/j.ygyno.2019.10.014.

45. Zang, R. et al. Pazopanib (Paz) monotherapy in Asian women who have not progressed after firstline chemotherapy for advanced ovarian, Fallopian tube, or primary peritoneal carcinoma. J. Clin. Oncol. 31, 5512-5512 (2013).

46. Vergote, I. et al. Trebananib or placebo plus carboplatin and paclitaxel as first-line treatment for advanced ovarian cancer (TRINOVA-3/ENGOT-ov2/GOG-3001): a randomised, double-blind, phase 3 trial. Lancet Oncol. 20, 862-876 (2019).

47. Coleman, R. et al. Analysis of survivorship in high-risk patients on treated on GOG-218. Gynecol. Oncol. 130, e112-e113 (2013).

48. Ray-Coquard, I. et al. Final results from GCIG/ENGOT/AGO-OVAR 12, a randomised placebocontrolled phase III trial of nintedanib combined with chemotherapy for newly diagnosed advanced ovarian cancer. Int. J. Cancer 146, 439-448 (2020).

49. Oza, A. M. et al. Standard chemotherapy with or without bevacizumab for women with newly diagnosed ovarian cancer (ICON7): Overall survival results of a phase 3 randomised trial. Lancet Oncol. 16, 928-936 (2015).

50. Randall, L. et al. Outcome differences in patients with advanced epithelial ovarian, primary peritoneal and fallopian tube cancers treated with and without bevacizumab. Gynecol. Oncol. 130, e33e34 (2013).

51. Shi, Q. \& Sargent, D. J. Meta-analysis for the evaluation of surrogate endpoints in cancer clinical trials. International Journal of Clinical Oncology vol. 14 102-111 (2009).

52. Kay, A., Higgins, J., Day, A. G., Meyer, R. M. \& Booth, C. M. Randomized controlled trials in the era of molecular oncology: Methodology, biomarkers, and end points. Ann. Oncol. 23, 1646-1651 (2012).

53. S, D., P, T. \& A, C. A Review of Studies Examining the Relationship between Progression-Free Survival and Overall Survival in Advanced or Metastatic Cancer. A Review of Studies Examining the Relationship between Progression-Free Survival and Overall Survival in Advanced or Metastatic Cancer (2012).

54. Kim, C. \& Prasad, V. Cancer drugs approved on the basis of a surrogate end point and subsequent overall survival: An analysis of 5 years of us food and drug administration approvals. JAMA Internal 
Medicine vol. 175 1992-1994 (2015).

55. Pasalic, D. et al. Progression-free survival is a suboptimal predictor for overall survival among metastatic solid tumour clinical trials. Eur. J. Cancer 136, 176-185 (2020).

56. Prasad, V., Kim, C., Burotto, M. \& Vandross, A. The strength of association between surrogate end points and survival in oncology: A systematic review of trial-level meta-analyses. JAMA Intern. Med. 175, 1389-1398 (2015).

57. Paoletti, X. et al. Assessment of Progression-Free Survival as a Surrogate End Point of Overall Survival in First-Line Treatment of Ovarian Cancer: A Systematic Review and Meta-analysis. JAMA Netw. open 3, e1918939 (2020).

58. Bentink, S. et al. Angiogenic mRNA and microRNA gene expression signature predicts a novel subtype of serous ovarian cancer. PLoS One 7, (2012).

59. Backen, A. et al. The combination of circulating Ang1 and Tie2 levels predicts progression-free survival advantage in bevacizumab-treated patients with ovarian cancer. Clin. Cancer Res. 20, 45494558 (2014).

60. Gourley, C. et al. Molecular subgroup of high-grade serous ovarian cancer (HGSOC) as a predictor of outcome following bevacizumab. | 2014 ASCO Annual Meeting | Abstracts | Meeting Library. J. Clin. Oncol. 32, Abstract 5502 (2014).

61. Collinson, F. et al. Predicting response to bevacizumab in ovarian cancer: A panel of potential biomarkers informing treatment selection. Clin. Cancer Res. 19, 5227-5239 (2013).

62. Michael J. Birrer, YounJeong Choi, Mark F. Brady, Robert S. Mannel, Robert Allen Burger, WEI WEl, Amreen Husain, Carlos Bais, NRG Oncology/Gynecologic Oncology Group; Massachusetts General Hospital/Dana Farber Cancer Center, Boston, MA; Genentech, Inc., S, M. Retrospective analysis of candidate predictive tumor biomarkers (BMs) for efficacy in the GOG-0218 trial evaluating front-line carboplatin-paclitaxel (CP) \pm bevacizumab (BEV) for epithelial ovarian cancer (EOC). J Clin Oncol 33, 2015 (suppl; abstr 5505).

63. Pignata, S. et al. Pazopanib plus weekly paclitaxel versus weekly paclitaxel alone for platinumresistant or platinum-refractory advanced ovarian cancer (MITO 11): A randomised, open-label, phase 2 trial. Lancet Oncol. 16, 561-568 (2015).

64. Chekerov, R. et al. Sorafenib plus topotecan versus placebo plus topotecan for platinum-resistant ovarian cancer (TRIAS): a multicentre, randomised, double-blind, placebo-controlled, phase 2 trial. Lancet Oncol. 19, 1247-1258 (2018).

65. Ray-Coquard, I. et al. Olaparib plus Bevacizumab as First-Line Maintenance in Ovarian Cancer. $N$. Engl. J. Med. (2019) doi:10.1056/nejmoa1911361. 


\section{Figures}

Fig 1a.

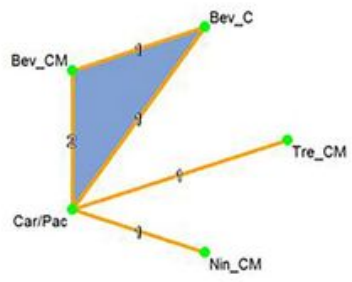

Fig 1b.

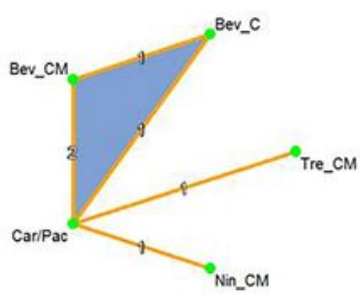

Fig 1c.

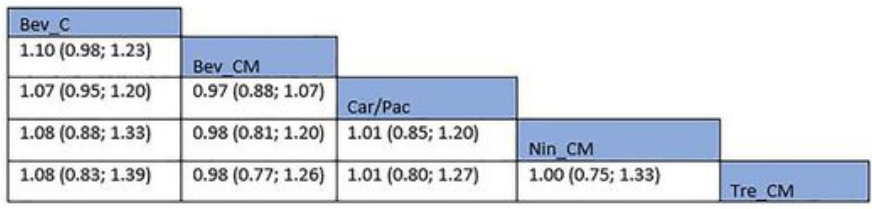

Fig 1 d.

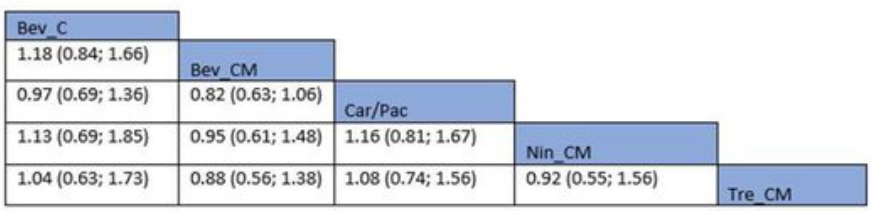

Fig 1e.

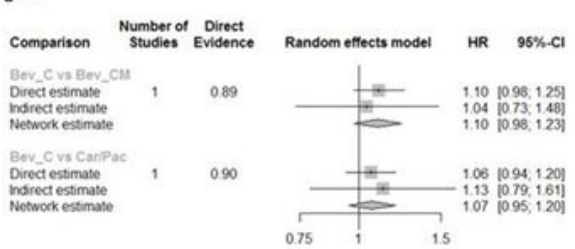

Fig 1f.

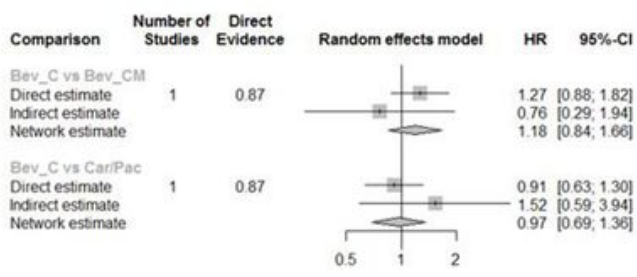

\section{Figure 1}

Fig 1a. Network plot of comparisons among different anti-angiogenic agents and standard of care chemotherapy (i.e. Carboplatin/Paclitaxel) for overall survival in chemotherapy naïve setting. Fig 1b. Network plot of comparisons among different anti-angiogenic agents and standard of care 
chemotherapy (i.e. Carboplatin/Paclitaxel) for progression-free survival in chemotherapy naïve setting. Fig 1c. Comparative effectiveness of different anti-angiogenic agents and standard of care chemotherapy (i.e. Carboplatin/Paclitaxel) for overall survival in chemotherapy naïve setting. Fig $1 \mathrm{~d}$. Comparative effectiveness of different anti-angiogenic agents and standard of care chemotherapy (i.e. Carboplatin/Paclitaxel) for progression-free survival in chemotherapy naïve setting. Fig 1e. Results based on the Separating Indirect from Direct Evidence (SIDE) approach to evaluating inconsistency in the network meta-analysis for overall survival in chemotherapy naïve setting. Key: Bev_C, Bevacizumab (concurrent); Bev_CM, Bevacizumab (concurrent + maintanence); Car/Pac, Carboplatin/Paclitaxel; Nin_CM, Nintedanib (concurrent + maintanence); Tre_CM, Trebananib (concurrent + maintanence). Fig $1 \mathrm{f}$. Results based on the Separating Indirect from Direct Evidence (SIDE) approach to evaluating inconsistency in the network meta-analysis for progression-free survival in chemotherapy naïve setting. Note: Nodes represent the interventions, lines connecting nodes represent direct comparisons between pairs of interventions, number stated on the lines represent the number of studies involved in the comparisons. Note: Values in each cell represents hazard ratio (HR and 95\% confidence interval) of the intervention at the top, compared to the comparator on the left. When $\mathrm{HR}<1$, prefers the column intervention, indicating that the column intervention is more effective than the row intervention on reducing overall survival. When $\mathrm{HR}>1$, prefers the row intervention. Significant results are in bold and underlined. Test for disagreement (direct versus indirect) for both comparisons: $p$-value $=0.75$. Key: Bev_C, Bevacizumab (concurrent); Bev_CM, Bevacizumab (concurrent + maintanence); Car/Pac, Carboplatin/Paclitaxel 


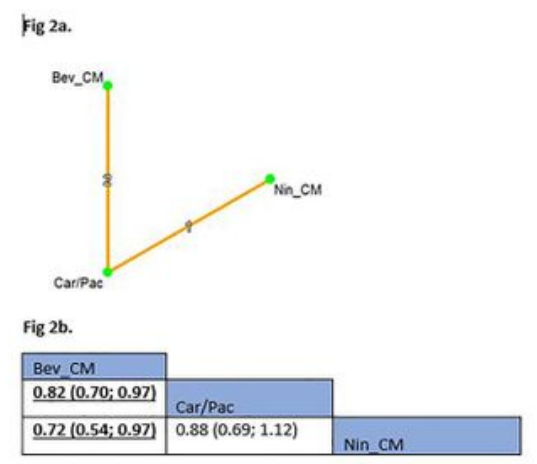

Fig 2c.

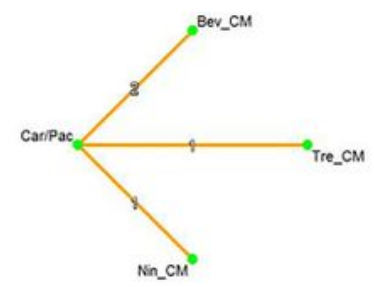

Fig 2d.
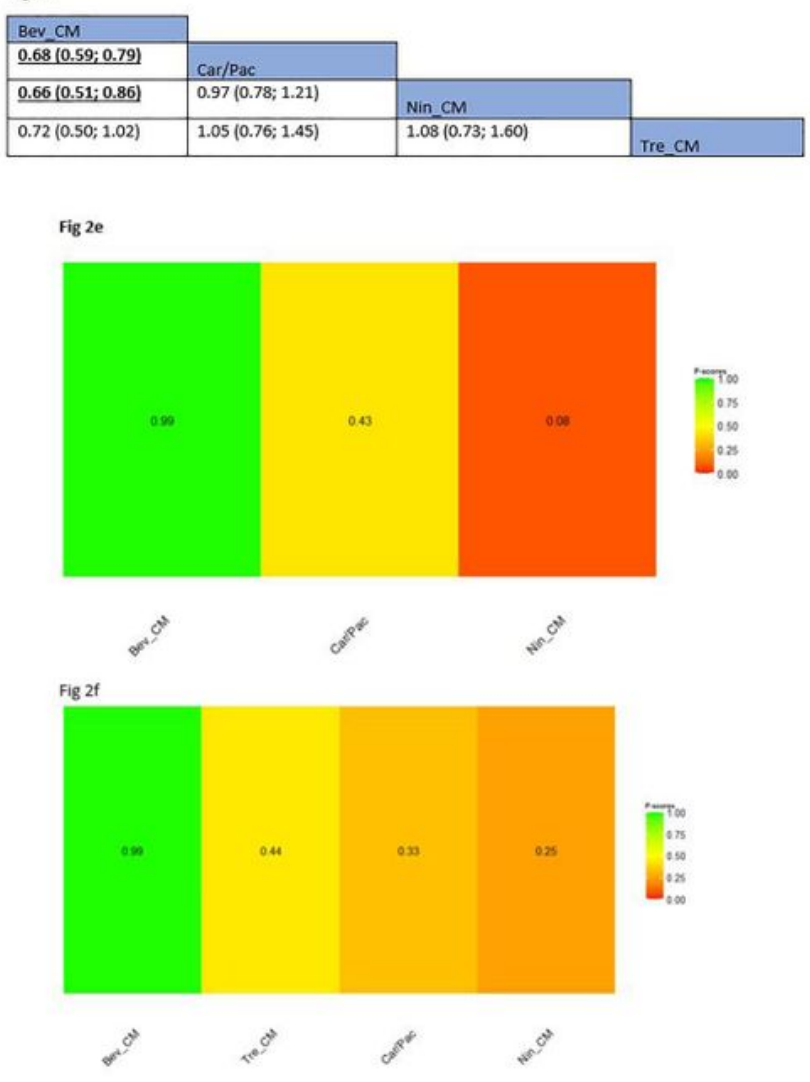

\section{Figure 2}

Fig 2a. Network plot of comparisons among different anti-angiogenic agents and standard of care chemotherapy (i.e. Carboplatin/Paclitaxel) for overall survival for high risk group in chemotherapy naïve setting. Fig $2 \mathrm{~b}$. Comparative effectiveness of different anti-angiogenic agents and standard of care chemotherapy (i.e. Carboplatin/Paclitaxel) for overall survival for high risk group in chemotherapy naïve setting. Fig 2c. Network plot of comparisons among different anti-angiogenic agents and standard of 
care chemotherapy (i.e. Carboplatin/Paclitaxel) for progression-free survival for high risk group in chemotherapy naïve setting. Fig $2 d$. Comparative effectiveness of different anti-angiogenic agents and standard of care chemotherapy (i.e. Carboplatin/Paclitaxel) for progression-free survival for high risk group in chemotherapy naïve setting. Fig 2e. Comparative effectiveness of different anti-angiogenic agents and standard of care chemotherapy (i.e. Carboplatin/Paclitaxel) for high risk group in chemotherapy naïve setting, P-scores for overall survival. Fig $2 f$. Comparative effectiveness of different anti-angiogenic agents and standard of care chemotherapy (i.e. Carboplatin/Paclitaxel) for high risk group in chemotherapy naïve setting, P-scores for progression-free survival. Key: Bev_CM, Bevacizumab (concurrent + maintanence); Car/Pac, Carboplatin/Paclitaxel; Nin_CM, Nintedanib (concurrent + maintanence). Note: Nodes represent the interventions, lines connecting nodes represent direct comparisons between pairs of interventions, number stated on the lines represent the number of studies involved in the comparisons. Note: Values in each cell represents hazard ratio (HR and 95\% confidence interval) of the intervention at the top, compared to the comparator on the left. When $H R<1$, prefers the column intervention, indicating that the column intervention is more effective than the row intervention on reducing overall survival. When $\mathrm{HR}>1$, prefers the row intervention. Significant results are in bold and underlined. The higher the P-scores, the higher likelihood that an intervention is in the top rank or one of the top ranks.

Fig 3a.
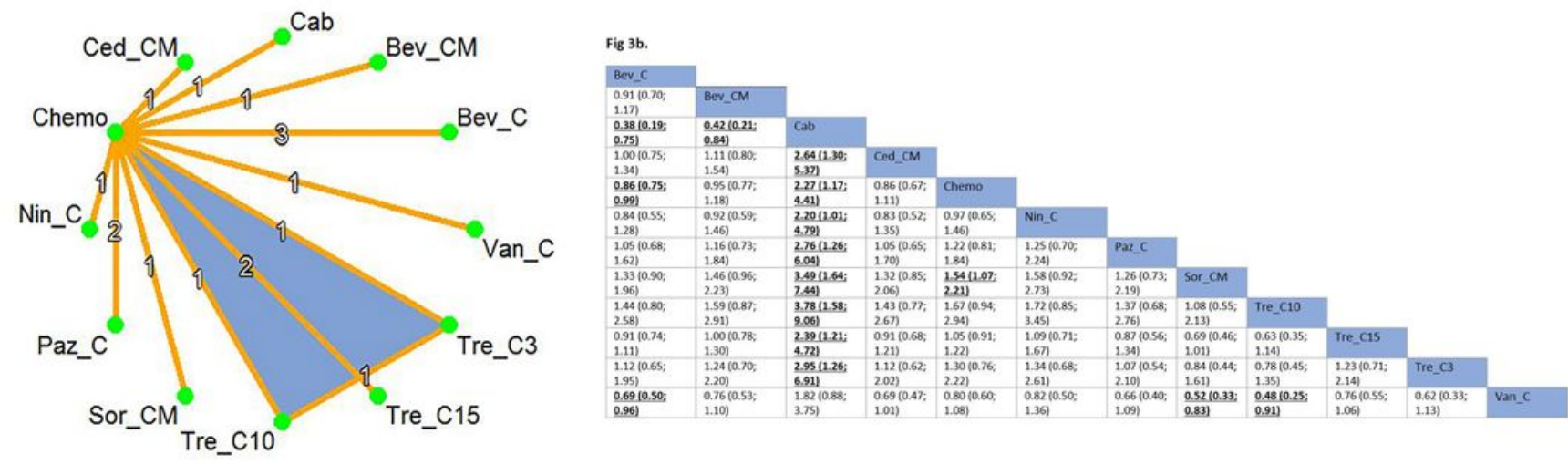

Fig 3c.
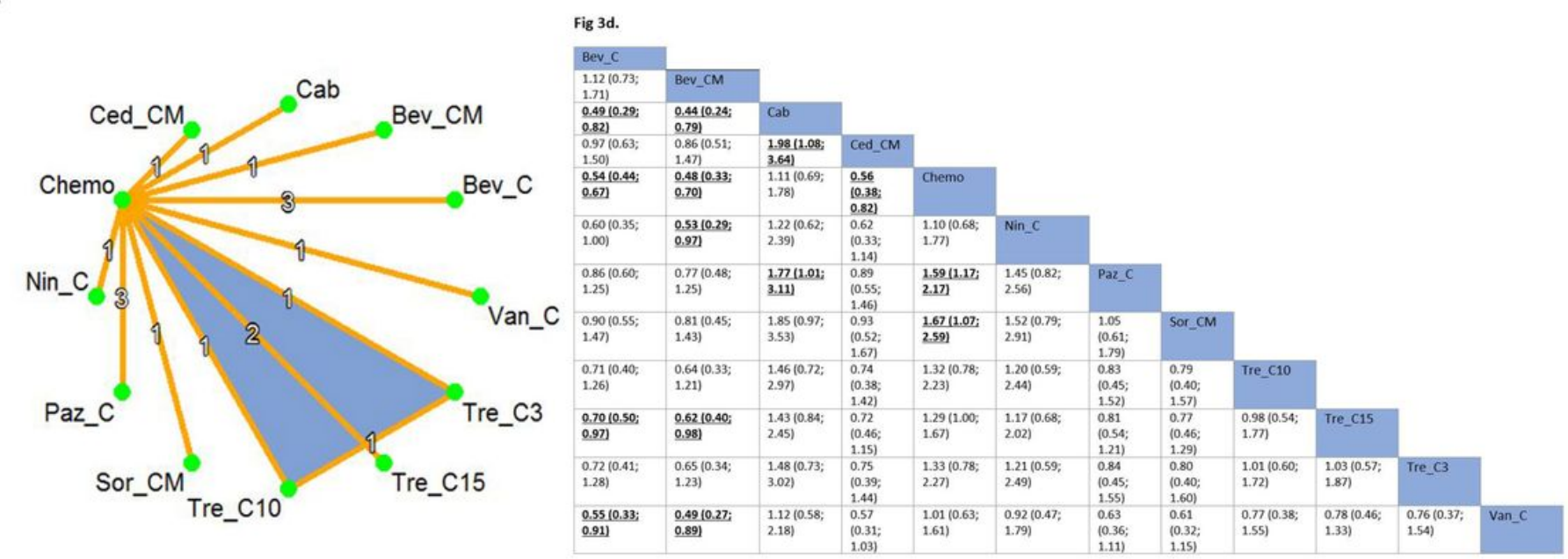


\section{Figure 3}

Fig 3a. Network plot of comparisons among different anti-angiogenic agents and standard of care chemotherapy for overall survival in recurrent EOC setting. Fig 3b. Comparative effectiveness of different anti-angiogenic agents and standard of care chemotherapy for overall survival in recurrent EOC setting. Fig 3c. Network plot of comparisons among different anti-angiogenic agents and standard of care chemotherapy for progression-free survival in recurrent EOC setting. Fig 3d. Comparative effectiveness of different anti-angiogenic agents and standard of care chemotherapy for progression-free survival in recurrent EOC setting. Fig 3e. Comparative effectiveness of different anti-angiogenic agents and standard of care chemotherapy in recurrent EOC setting, P-scores for overall survival. Fig 3f. Comparative effectiveness of different anti-angiogenic agents and standard of care chemotherapy in recurrent EOC setting, P-scores for progression-free survival. Key: Bev_C, Bevacizumab (concurrent); Bev_CM, Bevacizumab (concurrent + maintenance); Cab, Cabozantinib; Ced_CM, Cediranib (concurrent + maintenance); Chemo, Standard of care chemotherapy; Nin_C, Nintedanib (concurrent); Paz_C, Pazopanib (concurrent); Sor_CM, Sorafenib (concurrent + maintenance); Tre_C3, Trebananib (3mg/kg) (concurrent); Tre_C10, Trebananib (10mg/kg) (concurrent); Tre_C15, Trebananib $(15 \mathrm{mg} / \mathrm{kg})$ (concurrent); Van_C, Vandetanib (concurrent). Note: Nodes represent the interventions, lines connecting nodes represent direct comparisons between pairs of interventions, number stated on the lines represent the number of studies involved in the comparisons. Note: Values in each cell represents hazard ratio (HR and 95\% confidence interval) of the intervention at the top, compared to the comparator on the left. When $\mathrm{HR}<1$, prefers the column intervention, indicating that the column intervention is more effective than the row intervention on reducing overall survival. When $\mathrm{HR}>1$, prefers the row intervention. Significant results are in bold and underlined. The higher the P-scores, the higher likelihood that an intervention is in the top rank or one of the top ranks. 


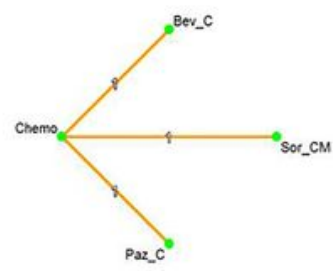

Fig 4b.

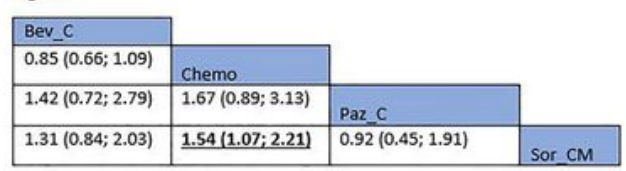

Fig $4 c$.

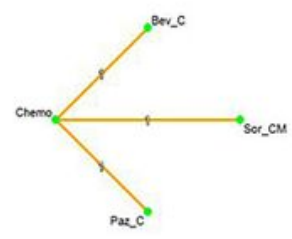

Fig 4d.

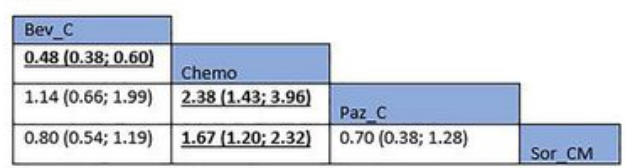

Fig 4 e.
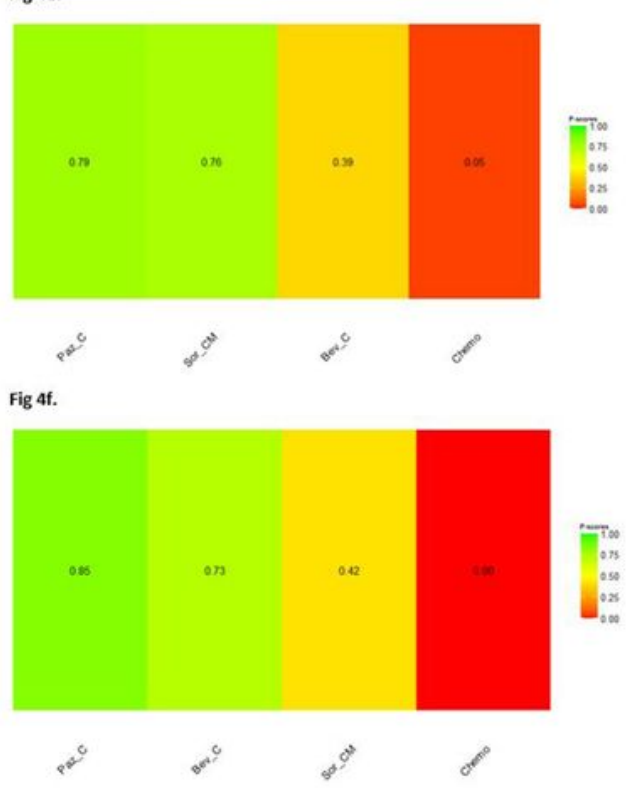

\section{Figure 4}

Fig 4a. Network plot of comparisons among different anti-angiogenic agents and standard of care chemotherapy for overall survival in platinum resistant setting. Fig 4b. Comparative effectiveness of different anti-angiogenic agents and standard of care chemotherapy for overall survival in platinum resistant setting. Fig 4c. Network plot of comparisons among different anti-angiogenic agents and standard of care chemotherapy for progression-free survival in platinum resistant setting. Fig 4d. 
Comparative effectiveness of different anti-angiogenic agents and standard of care chemotherapy for progression-free survival in platinum resistant setting. Fig 4e. Comparative effectiveness of different antiangiogenic agents and standard of care chemotherapy in platinum resistant setting, P-scores for overall survival. Fig 4f. Comparative effectiveness of different anti-angiogenic agents and standard of care chemotherapy in platinum resistant setting, P-scores for progression-free survival. Key: Bev_C, Bevacizumab (concurrent); Chemo, Standard of care chemotherapy; Paz_C, Pazopanib (concurrent); Sor_CM, Sorafenib (concurrent + maintenance). Note: Nodes represent the interventions, lines connecting nodes represent direct comparisons between pairs of interventions, number stated on the lines represent the number of studies involved in the comparisons. Note: Values in each cell represents hazard ratio (HR and $95 \%$ confidence interval) of the intervention at the top, compared to the comparator on the left. When $H R<1$, prefers the column intervention, indicating that the column intervention is more effective than the row intervention on reducing overall survival. When $\mathrm{HR}>1$, prefers the row intervention. Significant results are in bold and underlined. The higher the P-scores, the higher likelihood that an intervention is in the top rank or one of the top ranks. 
kig 5 .

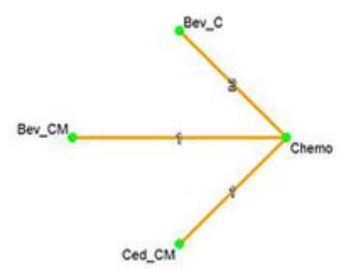

Fig 5b.

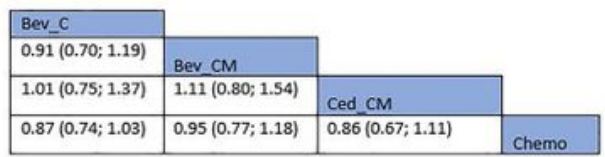

Fig 5c.

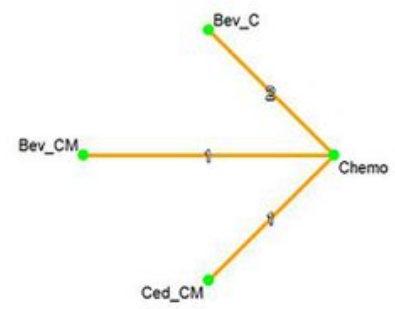

Fig 5d.

Bev_C

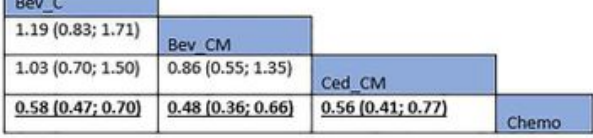

Fig se.

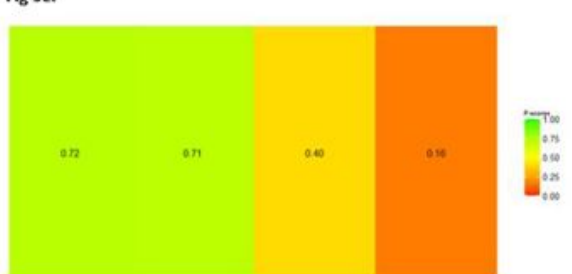

Fig $5 f$.
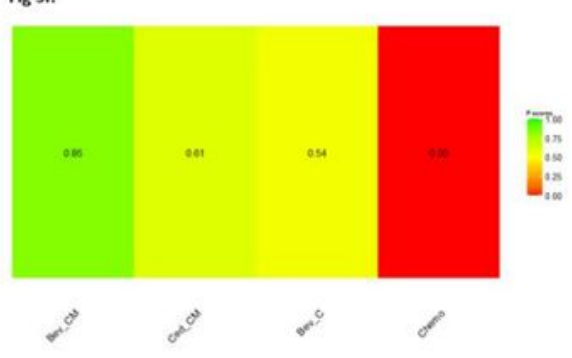

\section{Figure 5}

Fig 5a. Network plot of comparisons among different anti-angiogenic agents and standard of care chemotherapy for overall survival in platinum sensitive setting. Fig $5 \mathrm{~b}$. Comparative effectiveness of different anti-angiogenic agents and standard of care chemotherapy for overall survival in platinum sensitive setting. Fig 5c. Network plot of comparisons among different anti-angiogenic agents and standard of care chemotherapy for progression-free survival in platinum sensitive setting. Fig $5 d$. 
Comparative effectiveness of different anti-angiogenic agents and standard of care chemotherapy for progression-free survival in platinum sensitive setting. Fig $5 \mathrm{e}$. Comparative effectiveness of different antiangiogenic agents and standard of care chemotherapy in platinum sensitive setting, P-scores for overall survival. Fig $5 f$. Comparative effectiveness of different anti-angiogenic agents and standard of care chemotherapy in platinum sensitive setting, P-scores for progression-free survival. Key: Bev_C, Bevacizumab (concurrent); Bev_CM, Bevacizumab (concurrent + maintenance); Ced_CM, Cediranib (concurrent + maintenance); Chemo, Standard of care chemotherapy. Note: Nodes represent the interventions, lines connecting nodes represent direct comparisons between pairs of interventions, number stated on the lines represent the number of studies involved in the comparisons. Note: Values in each cell represents hazard ratio (HR and 95\% confidence interval) of the intervention at the top, compared to the comparator on the left. When $\mathrm{HR}<1$, prefers the column intervention, indicating that the column intervention is more effective than the row intervention on reducing overall survival. When $H R>1$, prefers the row intervention. Significant results are in bold and underlined. The higher the P-scores, the higher likelihood that an intervention is in the top rank or one of the top ranks.

\section{Supplementary Files}

This is a list of supplementary files associated with this preprint. Click to download.

- Supplementarydata.docx

- PRISMAabstractchecklist.docx

- PRISMAchecklist.docx 